\title{
Force and Oxygen Consumption in the Immature Rabbit Heart
}

\author{
MARK D. PARRISH AND SCOTT FARRAR \\ Department of Pediatrics, University of Texas Southwestern Medical Center, Dallas, Texas 75335
}

\begin{abstract}
We speculated that there are important agerelated differences in the economy of left ventricular force development in the isolated heart. To assess this, we evaluated oxygen consumption and force development in newborn (less than 1 wk old) $(n=26)$, juvenile $(4-6$ wk old) $(n=26)$, and adult (5-7 mo old) $(n=26)$ isolated, isovolumic rabbit hearts. Measurements were obtained with three different interventions, including 1 ) changes in heart rate, 2) inotropic stimulation with isoproterenol, and 3 ) changes in end-diastolic pressure. We found no significant baseline differences in the economy of force development. However, when heart rate was increased by $20 \%$, the force/oxygen consumption ratio (economy) increased in newborn hearts by approximately $37 \%$, whereas there was a decrease in juvenile and adult hearts of $\sim 27 \%$. In addition, with increases in end-diastolic pressure above $10 \mathrm{~mm}$ $\mathrm{Hg}$, newborn hearts increased their force/myocardial oxygen consumption ratio to $300 \%$ of the baseline value, whereas adults increased to only $160 \%$ of baseline. Isoproterenol produced no significant age-related differences in the force/myocardial oxygen consumption ratio. We conclude that there are important age-related differences in the economy of left ventricular force development in this model, but these differences are apparent only at higher heart rates and end-diastolic pressures. (Pediatr Res 27: 476-482, 1990)
\end{abstract}

\section{Abbreviations}

$\mathrm{dP} / \mathrm{dt}$, change in pressure per unit time

Little is known about developmental changes in myocardial oxygen consumption and the economy of force generation. Fisher et al. (1) found that newborn sheep had a higher myocardial oxygen consumption than adults, and related this increase to increased myocardial work. Suga et al. (2) studied the correlation between myocardial oxygen consumption and the pressure volume area in puppies. The slope of this correlation was not affected by contractile state or age. This suggests that myocardial mechanics (pressure-volume area) determine oxygen consumption and there are no fundamental changes in this relationship with maturation of the heart.

In contrast to these studies, theoretical considerations lead us to speculate that there may be important age-related changes in myocardial work economy unrelated to myocardial pressurevolume relationships. We know that the newborn myocardium may have different myosin isoenzymes than the adult $(3,4)$. Nakanishi et al. have shown in rabbits that the relative proportion

Received March 31, 1989; accepted January 2, 1990.

Correspondence and reprints: Mark D. Parrish, M.D., Division of Pediatric Cardiology, 2270 Stockton Blvd., Sacramento, CA 95817.

Supported in part by Grant $86 \mathrm{G}-087$ from the American Heart Association, Texas Affiliate. of myosin isozyme $V_{1}$ was greatest in the newborn and lowest in the adult (3). Furthermore, Kissling and Rupp (5) have demonstrated in rats that the degree of catecholamine-induced increase in myocardial oxygen consumption depends on the isoenzyme pattern of myosin. Perhaps the force/oxygen consumption ratio also changes with alterations in myocardial isoenzymes, although there are no data to confirm this.

We know that the newborn heart has a less mature sarcoplasmic reticulum than the adult. Mahony (6) demonstrated that there was a marked increase in the coupling of sarcoplasmic reticulum $\mathrm{Ca}^{2+}$ transport to ATP hydrolysis during maturation of the heart. In sheep, this maturational process seems to continue through the 8th wk of life. We speculated that this maturation of calcium coupling ratio might manifest as a developmental increase in the economy of myocardial force development.

Thus, there are fundamental biochemical differences between the newborn and adult myocardium. Differences in myosin isozyme or sarcoplasmic reticulum could conceivably alter the coupling of ATP hydrolysis to force generation. Therefore, we hypothesized that there might be important maturational changes in the economy of myocardial force generation independent of changes in ventricular pressure or volume. To further assess this, we evaluated oxygen consumption and force development in newborn, juvenile, and adult isolated, isovolumic rabbit hearts.

\section{MATERIALS AND METHODS}

Methods. Standard techniques for an isolated, isovolumic rabbit heart preparation were modified primarily to isolate and maintain the heart within the chest cavity (7). This was done to minimize both ischemic time and mechanical trauma to the heart. We used New Zealand White rabbits from three age groups: newborns (less than 1 wk old, mean age $3.4 \pm 2.0 \mathrm{~d})(n$ $=26)$, juveniles (4-6 wk old) $(n=26)$, and adults $(5-7$ mo old) $(n=26)$. Rabbits were sedated with pentobarbital $(50 \mathrm{mg} / \mathrm{kg})$ and ventilated. The pentobarbital was given i.v. to the juveniles and adults, and intraperitoneally to the newborns. A midline thoracotomy was performed, and the hearts were rapidly isolated and instrumented within the chest cavity. Retrograde coronary perfusion was begun with a warmed, oxygenated Krebs-Henseleit solution. Coronary perfusion was started without any intervening ischemic period. The perfusate had the following constituents $(\mathrm{mM})$ : glucose $4.9, \mathrm{NaCl} 110, \mathrm{NaHCO}_{3} 22, \mathrm{KCl} 3.4, \mathrm{MgSO}_{4}$, $0.5, \mathrm{CaCl}_{2}$ 1.8, and $\mathrm{KH}_{2} \mathrm{PO}_{4}$ 1.1.

Through a left atrial incision, a fluid-filled, balloon-tipped catheter was passed into the left ventricular cavity. The balloon was constructed from thin cellophane material. These balloons were of sufficient size that, when inflated with the maximum volume, the intrinsic balloon pressure was always less than 2 $\mathrm{mm} \mathrm{Hg}$. Before constructing the balloon, a probe was used to estimate the distance from the mitral valve annulus to the apex in each heart. Each balloon was tied to this length.

Coronary effluent was collected from the right ventricle with 
a soft catheter passed retrograde from the pulmonary artery. A 27-gauge thermistor needle was inserted superficially into the left ventricular muscle to monitor temperature. The myocardial temperature was maintained at $30^{\circ} \mathrm{C}$ throughout the experimental protocols by warming the perfusate and by external radiant warming of the chest cavity. Pacing wires were attached to the right atrium.

After instrumentation $(\sim 15 \mathrm{~min})$, hearts were allowed to stabilize for 30 to $60 \mathrm{~min}$. We noted no differences between the age groups in instrumentation or stabilization time. During this stabilization period, the pacing rate was set to at least $140 \mathrm{bpm}$, or $10 \%$ above the intrinsic heart rate. Fluid was added to the left ventricular balloon to adjust the ventricular diastolic pressure to approximately $8 \mathrm{~mm} \mathrm{Hg}$. Coronary perfusion rate was set at 1-3 $\mathrm{mL} / \mathrm{min}$ (newborn), $15-25 \mathrm{~mL} / \mathrm{min}$ (juvenile), and 40-60 $\mathrm{mL} / \mathrm{min}$ (adult) (7). These flow rates were chosen to give equivalent flow/g of tissue to all three age groups. Coronary effluent was discarded during the instrumentation and stabilization time period, with the expectation that this would allow sufficient washout of residual pentobarbital from the myocardium. In validation studies (7), this model was found to be stable for $3 \mathrm{~h}$ in all age groups, without tissue acidosis or evidence of lactate production.

Measurements and Calculations. After stabilization, the following measurements were obtained for all hearts: $l$ ) mean aortic pressure; 2) left ventricular diastolic pressure (corrected for intrinsic balloon pressure), developed pressure, and the first derivative of developed pressure; 3 ) coronary flow rate (measured effluent from right ventricle); 4 ) myocardial temperature; and 5) coronary arterial and venous perfusate gases. Perfusate was collected in glass syringes in a manner to avoid bubble contamination. The samples were kept on ice until the conclusion of the protocol when gas tensions and $\mathrm{pH}$ were assessed with a Radiometer blood gas apparatus (Radiometer, Westlake, $\mathrm{OH}$ ). All pressures were corrected for any baseline shifts due to manipulation of balloon volumes. Various protocols were then undertaken, as described below. At the end of each protocol, the hearts were arrested by adding potassium chloride to the perfusate $(20$ $\mathrm{mEq} / \mathrm{L}$ ). The fluid volume was removed from the balloon to decompress the heart. Repeat measurements were made in the arrested heart, including: 1) coronary flow rate, 2) myocardial temperature, and 3) coronary arterial and venous perfusate gases.

At the conclusion of each experiment, the left ventricle was opened and the shape and fit of each balloon in the cavity was inspected. The balloon length was measured and compared to the apical-mitral annulus distance. If the balloon was not of appropriate size for the ventricle, the data from the preparation were discarded. The fluid volume in the balloon was measured, and the preparation was discarded if there was any evidence of volume loss during the experiment. Atrial tissue was removed and discarded, the right ventricular free wall was dissected free from the left ventricle, and the two ventricular pieces were weighed separately (right ventricular free wall, and left ventricle with septum). The tissue pieces were then dried in an oven and reweighed.

Several calculations were made from our measurements. Arterial and venous oxygen contents were estimated from the oxygen tension:

$$
\text { oxygen content }(\mathrm{mL} / \mathrm{mL} \text { perfusate })=\mathrm{PO}_{2}(\text { torr }) \times 0.00003
$$

Myocardial oxygen consumption $\left(\mathrm{MVO}_{2}\right)$ was then calculated (8):

$$
\mathrm{MVO}_{2}=\left(\text { arterial } \mathrm{O}_{2} \text { content-venous } \mathrm{O}_{2} \text { content }\right) \times \text { coronary }
$$

The surface area of the left ventricular balloon was estimated, based upon the assumption that the balloon formed the shape of a prolate ellipsoid (9):

$$
\text { surface area }=2 \pi b H\left[\left(\sin ^{-1} a / H\right)+a\left(\left(H^{2}-a^{2}\right)^{0.5}\right) / H^{2}\right]
$$

where $\mathrm{a}=$ the short axis of the ellipsoid, $\mathrm{b}=$ the long axis of the ellipsoid, and $H=\left[1 /\left(a^{-4}\left(a^{2}-b^{2}\right)\right)\right]^{0.5}$. The long axis (a) of the balloon was directly measured and was constant throughout the experiment. However, the short axis (b) varied with the balloon volume, and was calculated from the following formula (9):

$$
\text { volume of a prolate ellipsoid }=(4 / 3) \pi a b^{2}
$$

Thus,

$$
\mathrm{b}=(\mathrm{Vol} / 4.187 \mathrm{a})^{0.5}
$$

The mean left ventricular pressure was assessed by measuring the area under the left ventricular systolic pressure curve. The systolic area for four beats was averaged and multiplied by the heart rate to give the mean systolic pressure/min. Inasmuch as no stroke work was performed in this model, the force generated by the left ventricle could be calculated (9):

mean force $/ \min$ (dynes) $=$

mean LV systolic pressure/min $\times$ balloon surface area $\times 1330$

The oxygen used for force development was estimated by subtracting the oxygen consumption after $\mathrm{KCl}$-arrest from the total oxygen consumption during each protocol. This was called the active oxygen consumption (active $\mathrm{MVO}_{2}$ ). The left ventricular economy was then estimated:

$$
\text { economy (dynes } / \mathrm{mL} \mathrm{O}_{2} \text { ) = mean force/active } \mathrm{MVO}_{2}
$$

Experimental Protocols. Several protocols were performed to study oxygen consumption and force development under various conditions.

Heart rate. The effects of altering heart rate on myocardial oxygen consumption and force development were assessed. After stabilization, hearts were paced at a rate of $140 \mathrm{bpm}$ for $10 \mathrm{~min}$. Measurements, as listed above, were performed. The pacing rate was then increased to $170 \mathrm{bpm}$, and after $10 \mathrm{~min}$, repeat measurements obtained. Eight hearts from each age group were studied by this protocol.

Inotropic stimulation. The effect of an isoproterenol infusion was studied. Paced heart rate was equal in all age groups for this protocol. After stabilization, baseline measurements were obtained. An isoproterenol infusion was then begun into the aortic root, with a delivered concentration of $0.02 \mu \mathrm{mol} / \mathrm{L}$. Because coronary flow/g was equal in all three age groups, the isoproterenol dose/g of tissue was equal in all groups. We have previously found that this dose produces a near maximal effect in our model with no apparent toxicity (10). After $10 \mathrm{~min}$ of drug infusion, repeat measurements were obtained. Ten hearts from each age group were studied by this protocol.

End-diastolic pressure. The effect of varying the balloon volume and end-diastolic pressure was assessed. Paced heart rate was equal in all age groups for this protocol. After stabilization, the balloon volume was reduced to achieve a left ventricular enddiastolic pressure of approximately $1 \mathrm{~mm} \mathrm{Hg}$. Every 5 to $10 \mathrm{~min}$, volume was added to the balloon to obtain measurements at four increments of left ventricular end-diastolic pressure $(1,3,6$, and $14 \mathrm{~mm} \mathrm{Hg}$ ). Eight hearts from each age group were studied by this protocol.

Statistical Analysis. Comparisons between independent groups (each age group) were performed using Bartlett's test for equality of variances, one-way analysis of variance, and Welch's approximation to the one-way analysis of variance for groups with unequal variances. In addition, Newman-Keuls multiple comparisons test was performed. For repeated measures, parametric repeated measures analysis of variance and multiple pairwise comparisons tests were performed. The level of statistical significance for all comparisons was chosen as $p<0.05(11)$.

\section{RESULTS}

Baseline and postprotocol measurements. Baseline measurements are summarized in Table 1. By design, heart rate, coronary 
Table 1. Baseline measurements (mean \pm SEM)

\begin{tabular}{|c|c|c|c|c|c|c|}
\hline & $\begin{array}{l}\text { Heart } \\
\text { rate } \\
(\mathrm{bpm})\end{array}$ & $\begin{array}{c}\text { Coronary } \\
\text { flow } / \mathrm{g} \\
\text { (mL/min) }\end{array}$ & $\begin{array}{c}\text { End-diastolic } \\
\text { pressure } \\
(\mathrm{mm} \mathrm{Hg})\end{array}$ & $\begin{array}{c}\text { Developed } \\
\text { pressure } \\
(\mathrm{mm} \mathrm{Hg})\end{array}$ & $\begin{array}{c}\text { Maximum } \\
+\mathrm{dP} / \mathrm{dt} \\
(\mathrm{mm} \mathrm{Hg} / \mathrm{s})\end{array}$ & $\begin{array}{c}\text { Total } \\
\mathrm{MVO}_{2} / \mathrm{g} \\
\left(\mathrm{mL} \cdot \mathrm{min}^{-1} \cdot \mathrm{g}^{-1}\right) \ddagger\end{array}$ \\
\hline Newborn & $141 \pm 0.8$ & $10.3 \pm 0.8$ & $9.2 \pm 0.8$ & $22 \pm 2.5^{*} \dagger$ & $218 \pm 23^{*} \dagger$ & $0.073 \pm 0.006^{*} \dagger$ \\
\hline Juvenile & $142 \pm 0.7$ & $9.9 \pm 0.7$ & $9.8 \pm 0.5$ & $41 \pm 2.5$ & $359 \pm 22$ & $0.056 \pm 0.003^{*}$ \\
\hline Adult & $142 \pm 0.8$ & $8.7 \pm 0.5$ & $9.9 \pm 0.9$ & $43 \pm 3.4$ & $396 \pm 37$ & $0.040 \pm 0.003 \dagger$ \\
\hline
\end{tabular}

* different from adult group, $p<0.05$.

$\dagger$ different from juvenile group, $p<0.05$.

$+\mathrm{MVO}_{2}$, myocardial oxygen consumption.

flow/g of tissue, and end-diastolic pressure were not significantly different between the age groups. Smaller hearts received a lower absolute flow rate and had a lower coronary resistance, producing a significantly lower perfusion pressure in newborns compared with juveniles and adults $(48,77$, and $102 \mathrm{~mm} \mathrm{Hg}$ in newborns, juveniles, and adults, respectively, $p<0.05$ ). Perfusate $\mathrm{pH}$ and $\mathrm{PCO}_{2}$ (not shown in Table 1) were not different between groups (newborn perfusate $\mathrm{pH}=7.61 \pm 0.01, \mathrm{PCO}_{2}=22 \pm 0.4$; juvenile $\mathrm{pH}=7.63 \pm 0.01, \mathrm{PCO}_{2}=20 \pm 0.7$; adult $\mathrm{pH}=7.65 \pm 0.01$, $\mathrm{PCO}_{2}=20 \pm 0.9$; values $=$ mean $\left.\pm \mathrm{SEM}\right)$. Perfusate $\mathrm{PO}_{2}$ differed slightly between groups (newborn $=545 \pm 10$ torr, juvenile $=$ $540 \pm 9$ torr, and adult $=513 \pm 8$ torr; $p<0.05$, with adults significantly different from juveniles and newborns). However, the pulmonary effluent $\mathrm{PO}_{2}$ was not significantly different between the age groups $(316,341$, and 328 torr in newborns, juveniles, and adults, respectively). The left ventricular-developed pressure and the first derivative of developed pressure were significantly lower in the newborn hearts than in the juveniles and adults. The total oxygen consumption/g of tissue was significantly higher in the newborns than in the juveniles and adults.

Table 2 displays some of the measurements obtained at the conclusion of the experimental protocols. We have previously described similar age-related differences in this model (7). Newborn hearts are about 1/30th the size of adult hearts, and 1/10th the size of juvenile hearts. As expected (12), newborn hearts have a relatively larger right ventricular mass. Newborn hearts also have a slightly lower water content in this model. After potassium chloride arrest, the oxygen consumption/g of tissue was higher in newborns than in the other age groups.

Figure 1 shows the baseline left ventricular force in our three age groups. There was a significant age-related increase in left ventricular force/min. Also shown in Figure 1 is the active myocardial oxygen consumption $/ \mathrm{min}$. We derived this value by subtracting the postarrest oxygen consumption from the total oxygen consumption. The active oxygen consumption (not corrected for tissue wt) also showed an age-related increase. Finally, Figure 1 displays the baseline left ventricular economy, which is defined as the force/min divided by the oxygen consumption/ min. There were no baseline differences between the age groups in this index of left ventricular economy.

Protocol results. Figure 2 illustrates the results of the first protocol, in which the hearts were assessed at two different pacing rates. With a $20 \%$ increase in pacing rate, active oxygen consumption in juvenile and adult hearts was $136 \pm 16 \%$ (mean \pm SEM) (not significantly different compared to baseline, $p=0.17$ ) and $132 \pm 12 \%(p<0.05$ compared to baseline), respectively. With the increase in heart rate, the oxygen consumption in newborn hearts was $84 \pm 16 \%$ of baseline (not significantly different from baseline, $p=0.12$ ). End-diastolic pressure went up to $179 \pm 16$ and $182 \pm 23 \%$ of baseline at the faster heart rate in juveniles and adults, respectively $(p<0.05$ compared to baseline). End-diastolic pressure did not change in newborns. The faster heart rate produced a small but significant decrease in left ventricular force per min and maximum positive $\mathrm{dP} / \mathrm{dt}$ in all of the age groups.

Figure 3 illustrates the results of the second protocol, in which isoproterenol was infused into the aortic root. With this dose, there was no significant change in heart rate in any of the age groups, with most hearts maintaining a paced rhythm. As shown, myocardial oxygen consumption, left ventricular force per min, and $\mathrm{dP} / \mathrm{dt}$ increased similarly in juvenile and adult groups $(p<$ 0.05 compared to baseline). However, the newborn group had a minimal response to this drug infusion although the changes from baseline in left ventricular force and ${ }^{+} \mathrm{dP} / \mathrm{dt}$ were significant. There was a large variability in the end-diastolic pressure with the drug infusion, and no significant differences were detected between the age groups.

Figure 4 shows the response of the hearts to an increase in end-diastolic pressure (protocol 3). Increasing end-diastolic pressure from 1 to $14 \mathrm{~mm} \mathrm{Hg}$ led to a $15-20 \%$ increase in myocardial oxygen consumption in juvenile hearts $(p<0.05)$. Although the changes appeared similar in the adult group, the differences from baseline were not significant. Left ventricular force increased to $180-190 \%$ of baseline in newborns and adults, whereas juveniles had a much greater increase to $\sim 290 \%$ of baseline $(p<0.05$ for all groups compared to baseline). $\mathrm{dP} / \mathrm{dt}$ increased significantly in all groups ( $p<0.05$ compared to baseline), but newborns showed significantly less increase than juveniles or adults.

Figure 5 summarizes the changes in left ventricular economy with each of the three protocols. With a $20 \%$ increase in heart rate, the mean newborn force/myocardial oxygen consumption ratio was $37 \%$ higher ( $p=0.27$ compared to baseline), whereas the mean juvenile and adult ratio was $27 \%$ lower $(p=0.04$ for juveniles and $p=0.08$ for adults compared to baseline). During isoproterenol infusion, economy appeared to increase slightly, but there was no difference between the age groups and no significant change from baseline. Changes in end-diastolic pressure had the greatest effect on the force/myocardial oxygen consumption ratio. In what we consider the normal range of end-diastolic pressures $(4-10 \mathrm{~mm} \mathrm{Hg}$, mean $=6.4 \mathrm{~mm} \mathrm{Hg}, \mathrm{SD}$

Table 2. Postprotocol measurements (mean \pm SEM)

\begin{tabular}{|c|c|c|c|c|c|c|}
\hline & $\begin{array}{l}\text { LV Wt } \\
(\mathrm{g}) \ddagger\end{array}$ & $\begin{array}{c}\mathrm{LV} / \mathrm{RV} \text { wt } \\
\text { (ratio) }\end{array}$ & $\begin{array}{c}\text { LV wet/dry } \\
\text { wt } \\
\text { (ratio) }\end{array}$ & $\begin{array}{c}\text { Balloon } \\
\text { volume } \\
(\mathrm{mL})\end{array}$ & $\begin{array}{c}\text { Balloon } \\
\text { surface area } \\
\left(\mathrm{cm}^{2}\right)\end{array}$ & $\begin{array}{c}\text { Post- } \mathrm{KCl} \\
\mathrm{MVO}_{2} \\
\left(\mathrm{~mL} \cdot \mathrm{min}^{-1} \cdot \mathrm{g}^{-1}\right)\end{array}$ \\
\hline Newborn & $0.25 \pm 0.02^{* \dagger}$ & $2.90 \pm 0.14^{*} \dagger$ & $5.33 \pm 0.10^{* \dagger}$ & $0.039 \pm 0.005^{*} \dagger$ & $0.90 \pm 0.07^{*+}$ & $0.034 \pm 0.004 * \dot{f}$ \\
\hline Juvenile & $2.94 \pm 0.12 *$ & $3.46 \pm 0.08$ & $6.80 \pm 0.10$ & $0.261 \pm 0.023^{*}$ & $3.31 \pm 0.17^{*}$ & $0.022 \pm 0.002$ \\
\hline Adult & $7.23 \pm 0.24 \dagger$ & $3.23 \pm 0.11$ & $6.56 \pm 0.10$ & $0.839 \pm 0.079 \dagger$ & $7.30 \pm 0.43 \dagger$ & $0.0155 \pm 0.002$ \\
\hline
\end{tabular}

* different from adult group, $p<0.05$.

$\dagger$ different from juvenile group, $p<0.05$.

$\ddagger \mathrm{LV}$, left ventricle; $\mathrm{RV}$, right ventricle; $\mathrm{MVO}_{2}$, myocardial oxygen consumption. 


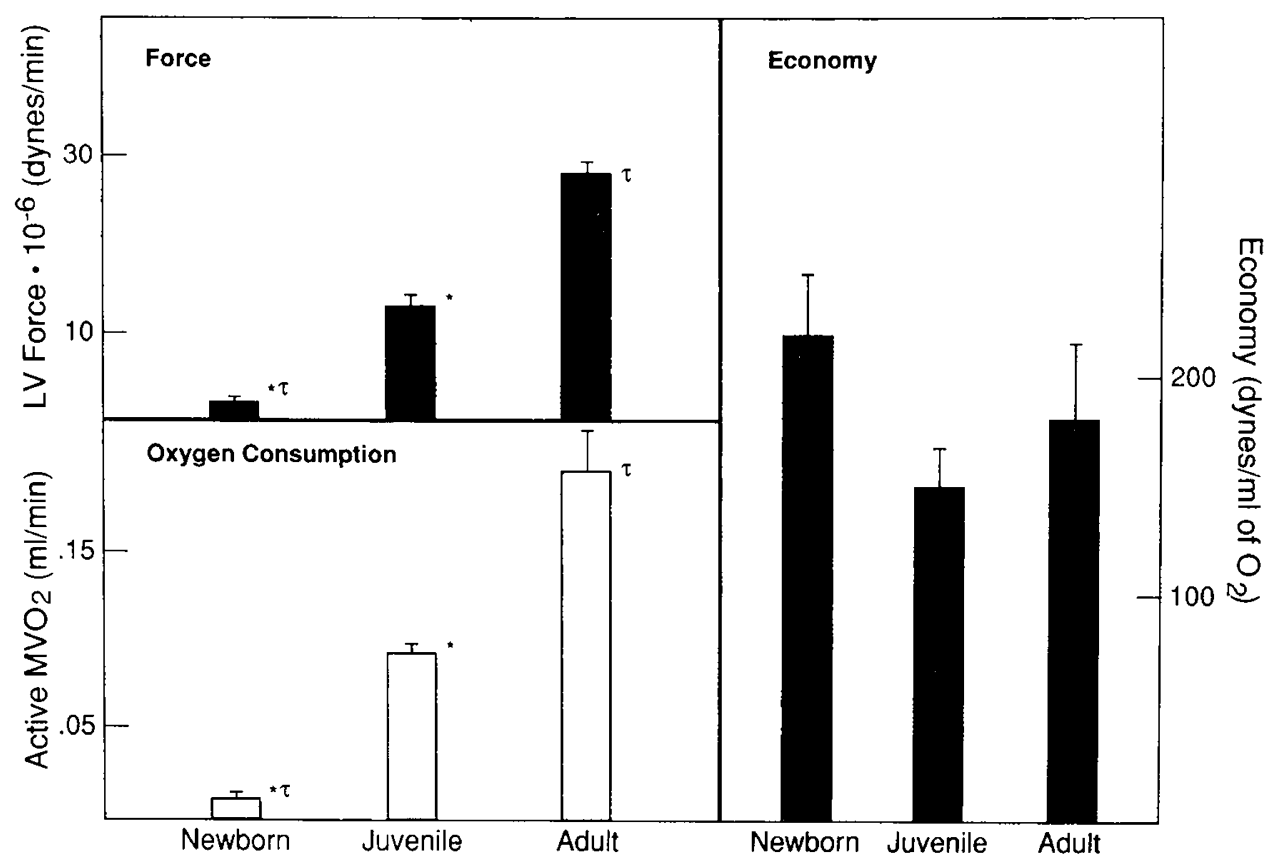

Fig. 1. Baseline myocardial force, oxygen consumption, and economy. Baseline myocardial force, oxygen consumption, and economy are displayed for the three age groups. Force is defined in the text. Active oxygen consumption is derived by subtracting the postarrest myocardial oxygen consumption from the total oxygen consumption. Left ventricular economy is defined as the force/min divided by the active oxygen consumption $/ \mathrm{min}$. Values are shown as means $\pm \mathrm{SEM} . \mathrm{MVO}_{2}$, myocardial oxygen consumption; LV, left ventricle. *, Significantly different from adult group, $p<0.05, \tau$, significantly different from juvenile group, $p<0.05$.

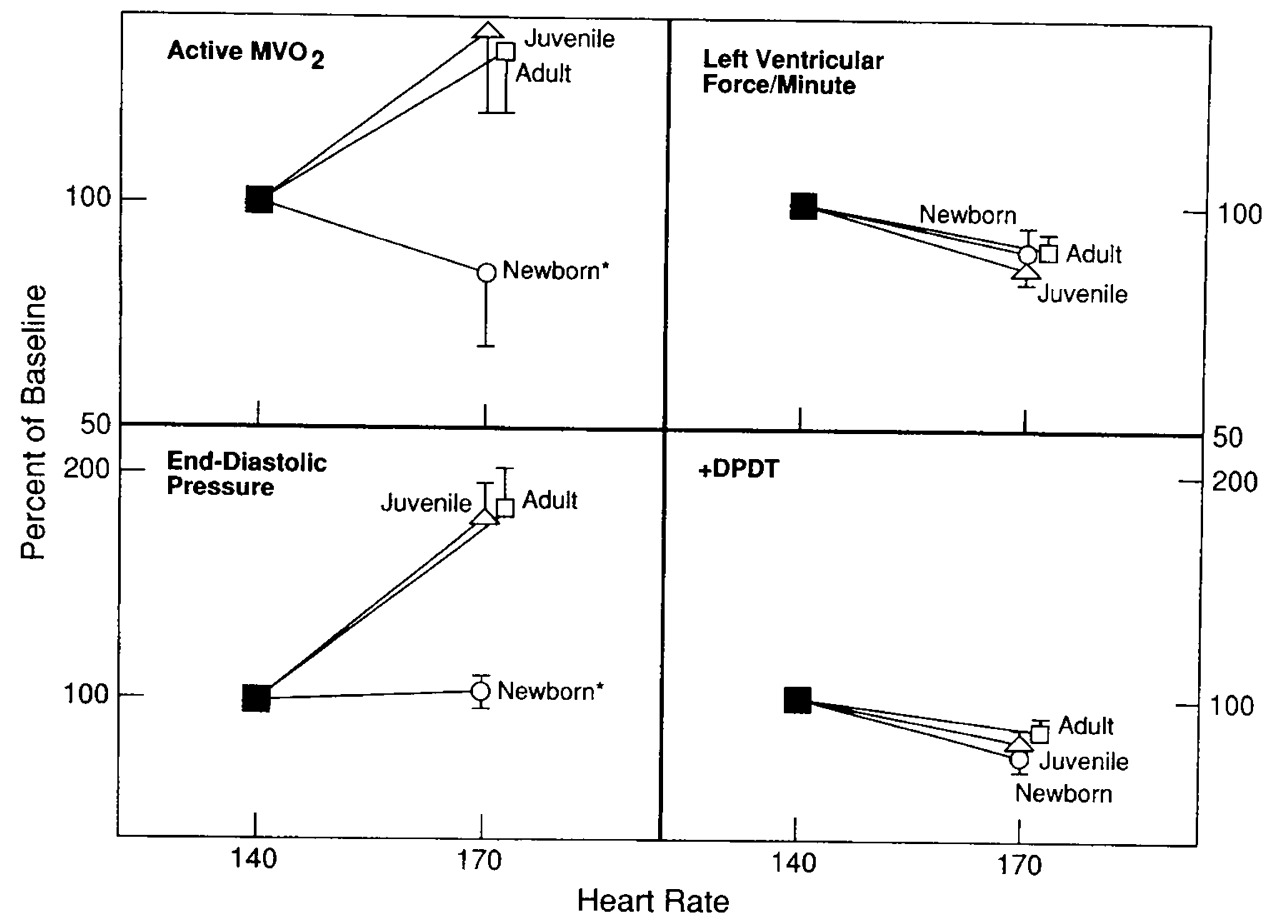

Fig. 2. Protocol 1-effect of increasing heart rate. The effects of heart rate change on active myocardial oxygen consumption, end-diastolic pressure, left ventricular force, and maximum positive DPDT are shown for the three age groups. Results at a heart rate of 170 bpm are shown as a percentage of the baseline values (heart rate $140 \mathrm{bpm}$ ). Values are shown as means $\pm \mathrm{SEM} . \mathrm{MVO}_{2}$, myocardial oxygen consumption. *, Significantly different from the adult and juvenile groups, $p<0.05$.

$= \pm 1.3 \mathrm{~mm} \mathrm{Hg}$ ), the mean economy was approximately $49 \%$ greater than baseline in all three age groups. This change from baseline was significant $(p<0.05)$ for the adult and juvenile groups only. At higher end-diastolic pressures, the newborn hearts increased their force/myocardial oxygen consumption ratio to $300 \%$ of baseline, which was significantly greater than the adults.

\section{DISCUSSION}

Our results suggest that there are age-related differences in the economy of oxygen utilization. Within the narrow bounds of our model, there were no significant baseline differences in left ventricular economy between newborn, juvenile, and adult hearts. However, with increasing heart rate, newborns had a 


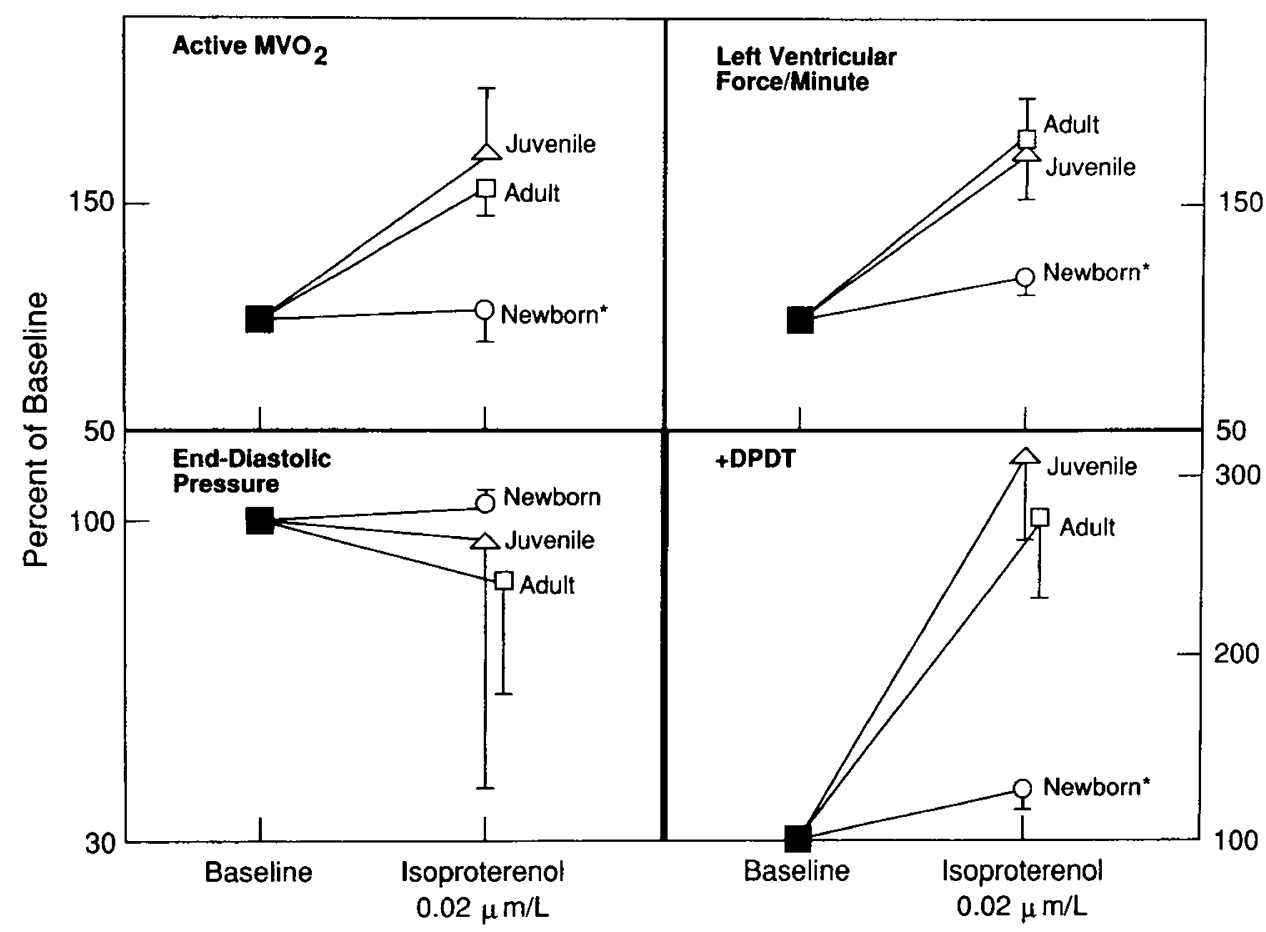

Fig. 3. Protocol 2-effect of isoproterenol. The effects of an isoproterenol infusion on active myocardial oxygen consumption, end-diastolic pressure, left ventricular force, and maximum positive DPDT are shown for the three age groups. Results at an isoproterenol dose of $0.05 \mu \mathrm{g} / \mathrm{mL}$ perfusate are shown as a percentage of the preceeding baseline values. Values are shown as means $\pm \mathrm{SEM} . \mathrm{MVO}_{2}$, myocardial oxygen consumption. *, Significantly different from the adult and juvenile groups, $p<0.05$.

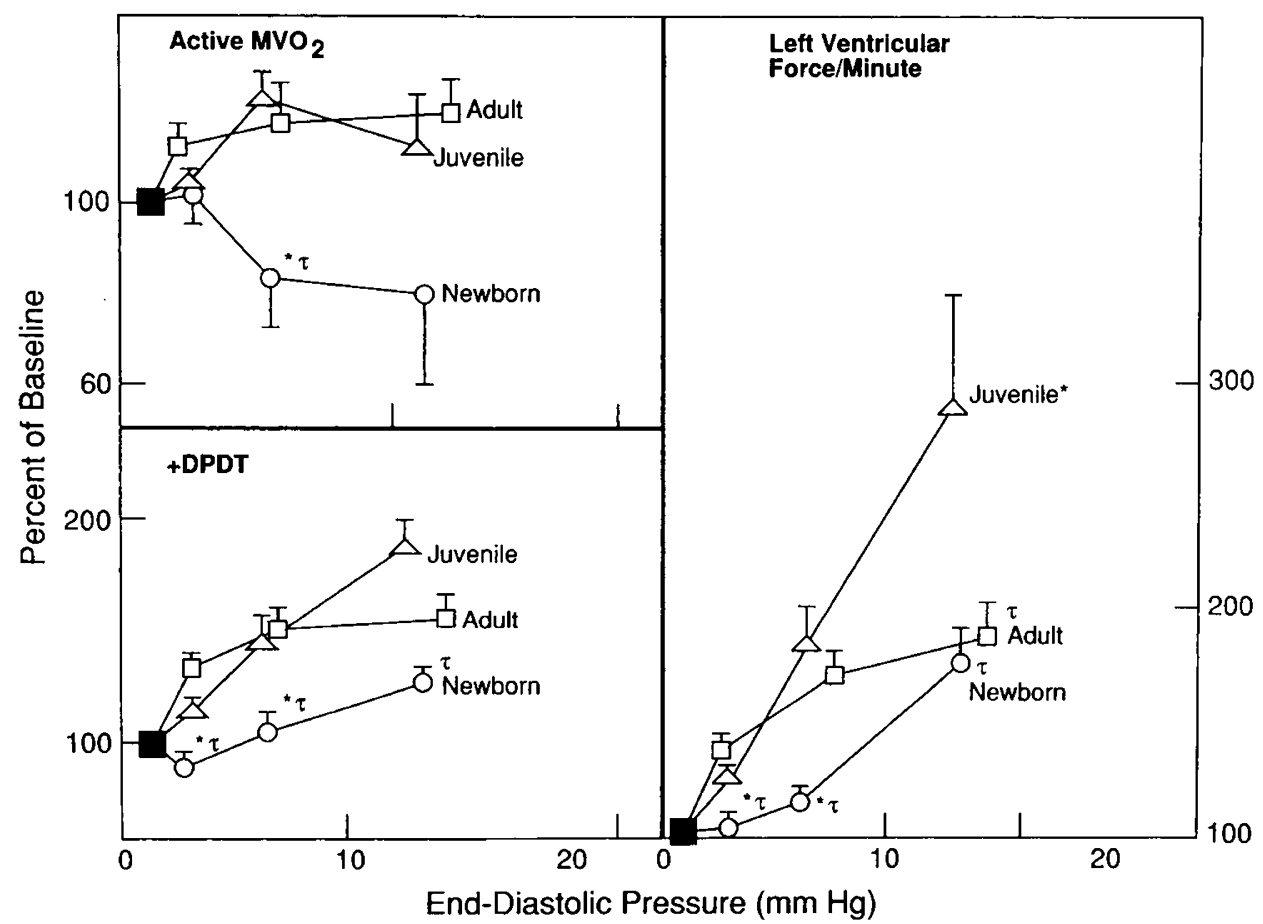

Fig. 4. Protocol 3-effect of changes in end-diastolic pressure. The effects of increasing end-diastolic pressure from 1 to $14 \mathrm{~mm} \mathrm{Hg}$ on active myocardial oxygen consumption, left ventricular force, and maximum positive DPDT are shown for the three age groups. Results at three incremental increases in balloon volume are shown as a percentage of the initial baseline values. Values are shown as means $\pm \mathrm{SEM} \mathrm{MVO}_{2}$, myocardial oxygen consumption. *, Significantly different from the adult group; $\tau$, significantly different from the juvenile group, $p<0.05$.

significant increase in the force/myocardial oxygen consumption ratio compared to the other two groups. Similarly, newborns increased their force/myocardial oxygen consumption ratio to a greater degree at high end-diastolic pressures. Inotropic stimulation with isoproterenol produced no age-related changes in economy.
Working with a different model, Fisher et al. (1) found that newborn sheep have a higher myocardial oxygen consumption than adult sheep. They attributed this finding largely to differences in myocardial work, implying that economy was similar between the age groups. However, because estimates of left ventricular force are extremely difficult in the intact ejecting 


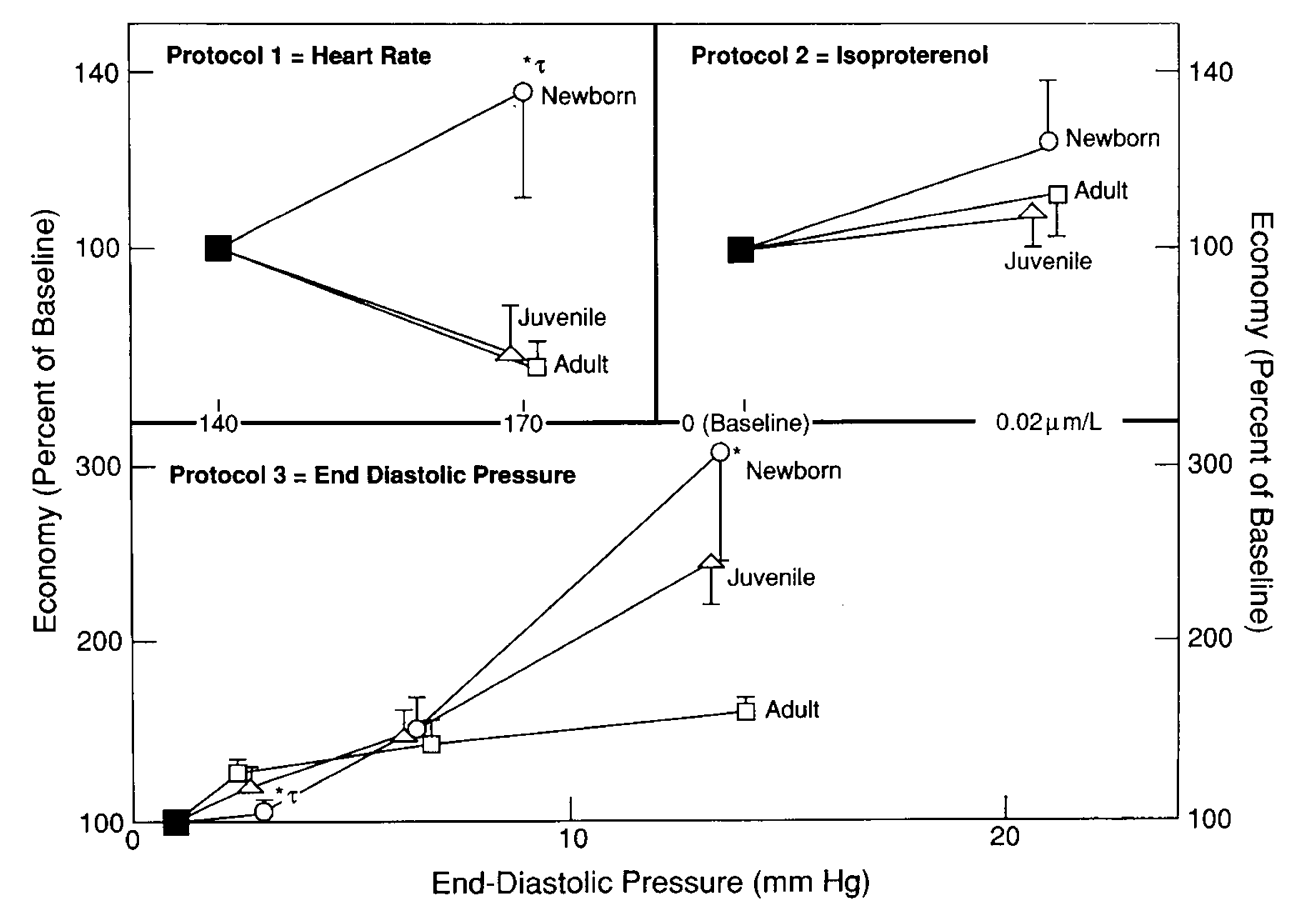

Fig. 5. Changes in left ventricular economy. The effects of heart rate, isoproterenol, and end-diastolic pressure on left ventricular economy are shown for the three age groups. Values are shown as a percentage of the initial baseline values, and are expressed as means \pm SEM. *, Significantly different from the adult group; $\tau$, significantly different from the juvenile group, $p<0.05$.

heart, small but significant age-related differences in myocardial economy might have remained undetected. Suga et al. (2) have related the left ventricular pressure-volume area to myocardial oxygen consumption in puppies. They found no significant differences between adult dogs and puppies (2-4 mo old), despite large differences in heart size and contractile properties. Our model is quite different from Suga et al.'s (2), yet we, too, found no baseline differences between the age groups when we related mechanics (pressure-volume relationship for Suga et al. (2) and force in our model) to oxygen consumption.

Several previously described features of the newborn heart might help to explain our results. First, as noted by Friedman (12), the newborn myocardium has a reduced compliance compared with the adult myocardium. This is associated with a greater noncontractile mass in the newborn heart. Perhaps similar preloading conditions in our model (similar end-diastolic pressures) produce quite different degrees of muscle fiber stretch because of age-related differences in noncontractile mass. Loiselle (13) has noted that the activation metabolism (energy needed for electrical excitation and excitation-contraction coupling) is altered by changing preload or muscle length. Thus, we speculate that age-related differences in myocardial compliance might produce age-related differences in myofilament alignment, which in turn might affect the activation metabolism of the heart. The need for a higher filling pressure to optimize myofilament alignment might explain our finding of improved economy of force production at high end-diastolic pressures in the newborn heart.

Second, the newborn myocardium has been noted to have an immature sarcoplasmic reticulum, and consequently has a greater reliance on the sarcolemma for excitation-contraction coupling (6). We speculate that differences between the two membranes in the coupling of ATP to calcium flux might produce differences in the efficiency of force development. For example, if calcium can transit the sarcolemma with less energy expenditure than transit across the sarcoplasmic reticulum, then the newborn heart (relying on transsarcolemmal flux) could produce a myocardial contraction more economically than the adult heart. If this speculation is valid, then our results at increased heart rates might be explained. If transsarcolemmal calcium flux were particularly economical, then the economy would be most easily noted at increased cycling rates.

In their baseline condition, newborn hearts appear to be operating at near maximal contractility, with very limited reserve compared to adults (14). It is possible that our interventions of increasing heart rate and increased end-diastolic pressure led to a decrease in contractility in the newborn hearts, but not in the adult hearts. This decreased contractility may have produced a decrease in oxygen consumption with very little change in left ventricular force.

Methodologic considerations may limit the applicability of our results beyond the bounds of this model. For example, glucose is the only metabolic substrate provided in our model. Because adult myocardium relies more on fatty acid oxidation than does neonate myocardium $(15,16)$, our model may place the adult heart at a relative disadvantage. Werner et al. (16) did note that 10-d-old rabbit hearts have an increased ability to oxidize palmitylCoA compared with the fetal heart. Their data suggest a fairly rapid maturational process for mitochondrial respiration. Although no information is available on the metabolic needs of 4- to 6-wk-old rabbit hearts, we speculate that our juveniles have substrate needs similar to those of the adult heart.

Our hearts were also hypothermic. Hypothermia has previously been noted to have no effect on left ventricular economy (17). However, there may be age-related differences in the effects of hypothermia on the myocardium. Several investigators have shown that the immature rabbit heart gains greater protection from ischemic injury with hypothermia than does the adult heart $(18,19)$. It is not clear how this protective effect of hypothermia would affect the economy of the myocardium as studied in our model.

It is possible that age-related differences in the response of the myocardium to the isolation process might account for some of our findings. For example, there may be age-related differences in the response of the heart to pentobarbital, although this drug should have been completely washed out from the myocardium before the experimental protocols began.

In summary, we found significant differences between adult and newborn hearts in left ventricular economy in an isolated, isovolumic rabbit heart model. However, these differences are 
apparent only at high heart rates and high end-diastolic pressures. Other workers using models similar to ours should consider these differences when making age-related comparisons of myocardial performance.

\section{REFERENCES}

1. Fisher DJ, Heymann MA, Rudolph AM 1981 Myocardial consumption of oxygen and carbohydrates in newborn sheep. Pediatr Res 15:843-846

2. Suga $\mathrm{H}$, Yamada $\mathrm{O}$, Goto $\mathrm{Y}$, Igarashi $\mathrm{Y}$, Yasumura $\mathrm{Y}$, Nozawa $\mathrm{T}$, Futaki $\mathrm{S}$ 1987 Left ventricular $\mathrm{O}_{2}$ consumption and pressure-volume area in puppies. Am J Physiol 253:H770-H776

3. Nakanishi T, Nagae M, Takao A 1986 Developmental changes in contractile protein adenosine 5 '-triphosphatase in the rabbit heart. Circ. Res 58:890895

4. Effron MB, Bhatnagar GM, Spurgeon HA, Ruanyo-Arroyo G, Lakatta EG 1987 Changes in myosin isoenzymes, ATPase activity, and contraction duration in rat cardiac muscle with aging can be modulated by thyroxine. Circ Res 60:238-245

5. Kissling G, Rupp H 1986 The influence of myosin isoenzyme pattern on increase in myocardial oxygen consumption induced by catecholamines. Basic Res Cardiol 81(suppl 1):103-115

6. Mahony L 1988 Maturation of calcium transport in cardiac sarcoplasmic reticulum. Pediatr Res 24:639-643

7. Parrish MD, Ayres NA, Kendrick BT, Fixler DE 1986 Maturational differences in the isolated isovolumic rabbit heart. Am J Physiol 251:H1143-H1148

8. Neely JR, Liebermeister H, Battersby EJ, Morgan HE 1967 Effect of pressure development on oxygen consumption by isolated rat heart. Am $\mathbf{J}$ Physiol 212:804-814

9. Smith CAB 1966 Biomathematics. Charles Griffen and Co., Ltd., New York, pp 295-305

10. Parrish MD, Brannon TS, Payne AE, Ayres NA, Fixler DE 1988 Nilrinone effects in the isolated immature rabbit heart. Dev Pharmacol Ther 11:328 337

11. Snedecor GW, Cochran WG 1967 Statistical Methods. Iowa State University Press, Ames, pp 1-500

12. Friedman WF 1973 The intrinsic physiologic properties of the developing heart. In: Sonnenblick EM (ed) Neonatal Heart Disease. Grune \& Stratton, New York, pp 21-49

13. Loiselle DS 1987 Cardiac basal and activation metabolism. Basic Res Cardiol 82(suppl 2):37-50

14. Teitel DF, Sidi D, Chin T, Brett C, Heymann MA, Rudolph AM 1985 Developmental changes in myocardial contractile reserve in the lamb. Pediatr Res 19:948-955

15. Oram JF, Bennetch SL, Neely JR 1973 Regulation of fatty acid utilization in isolated perfused rat hearts. J Biol Chem 248:5299-5309

16. Werner JC, Whitman V, Musselman J, Schuler HG 1982 Perinatal changes in mitochondrial respiration of the rabbit heart. Biol Neonate 42:208-216

17. Suga $H$, Goto $Y$, Igarashi $Y$, Yasumura $Y$, Nozawa T, Futaki S, Tanaka N 1988 Cardiac cooling increases $\mathrm{E}_{\max }$ without affecting relation between $\mathrm{O}_{2}$ consumption and systolic pressure-volume area in dog left ventricle. Circ Res 63:61-71

18. Bove EL, Stammers AH 1986 Recovery of left ventricular function after hypothermic global ischemia. J Thorac Cardiovasc Surg 91:1 15-122

19. Baker JE, Boerboom LE, Olinger GN 1988 Age-related changes in the ability of hypothermia and cardioplegia to protect ischemic rabbit myocardium. J Thorac Cardiovasc Surg 96:717-724 